\title{
EMOCIONES DE DOCENTES DE LA EDUCACIÓN MEDIA SUPERIOR ANTE LOS CAMBIOS DEL ENTORNO DURANTE EL CONFINAMIENTO POR EL COVID-19
}

\author{
María Esther Urrutia Aguilar \\ Maestría en Docencia para la Educación Media Superior \\ Facultad de Medicina, UNAM. \\ Silvia Ortiz León \\ Departamento de Psiquiatría y Salud Mental \\ Facultad de Medicina, UNAM \\ Aurora Leonila Jaimes Medrano \\ Departamento de Psiquiatría y Salud Mental, Facultad de Medicina, UNAM \\ aurojm@facmed.unam.mx
}

Recepción Artículo: 19 octubre 2020

Admisión Evaluación: 15 octubre 2020

Informe Evaluador 1: 25 noviembre 2020

Informe Evaluador 2: 23 noviembre 2020

Aprobación Publicación: 27 noviembre 2020

\section{RESUMEN}

Los estudios sobre brotes epidémicos generalmente reportan consecuencias negativas asociadas con el confinamiento y su impacto en la Salud Mental; en la educación es importante estudiar los efectos sobre todo en los docentes ya que, es un profesional que durante la pandemia tuvo que migrar de una modalidad presencial a una modalidad remota para impartir sus clases, generando en muchos estrés debido a que no contaban con la preparación suficiente para realizar esta tarea; además, en los docentes recae la responsabilidad de conducir los procesos de enseñanza y aprendizaje , por lo que deberán de fortalecer su resiliencia que puede ayudar a mitigar las repercusiones en los procesos cognitivos de los estudiantes. Por lo que este estudio tuvo como objetivo, identificar las emociones y reflexiones que experimentaron los profesores de bachillerato que se encontraban cursando, durante el confinamiento, la Maestría en Docencia para la Educación Media Superior, con el propósito de reconocer los componentes emocionales que participan en la construcción de la resiliencia frente a la adversidad. Se realizó un estudio exploratorio de corte transversal y analítico; a los participantes se les solicitó elegir un video y de ahí se establecieron las preguntas para la expresión de los sentimientos. El análisis de los resultados se llevó a cabo por medio del programa worditout (software gratis) que transforma el texto en nubes de palabras, se realizó un análisis bivariado utilizando el paquete estadístico SPSS versión 20. Las preguntas con respuesta abierta fueron analizadas desde la construcción de la narrativa. Los resultados arrojaron que los profesores mostraron en su mayoría emociones positivas ante la crisis generada por el COVID-19, que pudieron estar asociadas a la construcción de la resiliencia y que les ayuda a tener una perspectiva constructiva a pesar de la contingencia 0 adversidades.

Palabras clave: COVID-19; emociones; docentes; confinamiento; resiliencia. 


\title{
EMOCIONES DE DOCENTES DE LA EDUCACIÓN MEDIA SUPERIOR ANTE LOS CAMBIOS DEL ENTORNO DURANTE EL CONFINAMIENTO POR EL COVID-19
}

\begin{abstract}
Emotions of upper secondary education teachers in the face of changes in the environment during confinement due to COVID-19. Studies on epidemic outbreaks generally report negative consequences associated with confinement and its impact on Mental Health; In education, it is important to study the effects, especially on teachers, since it is a professional who during the pandemic had to migrate from a face-to-face mode to a remote mode to teach their classes, generating a lot of stress because they did not have the preparation enough to perform this task; Furthermore, teachers are responsible for conducting the teaching and learning processes, so they must strengthen their resilience that can help mitigate the repercussions in the cognitive processes of students. So this study aimed, identify the emotions and reflections experienced by the high school teachers who were studying, during confinement, the Master's Degree in Teaching for Higher Secondary Education, in order to recognize the emotional components that participate in building resilience in the face of adversity. An exploratory cross-sectional and analytical study was carried out; The participants were asked to choose a video and from there the questions for the expression of feelings were established. The analysis of the results was carried out by means of the worditout program (free software) that transforms the text into word clouds, a bivariate analysis was carried out using the statistical package SPSS version 20. The open-ended questions were analyzed from construction of the narrative. The results indicated that the

teachers showed mostly positive emotions in the face of the crisis generated by COVID-19, which could be associated with the construction of resilience and that helps them to have a constructive perspective despite the contingency or adversity.
\end{abstract}

Keywords: COVID-19; emotions; teachers; lockdown; resilience.

\section{INTRODUCCIÓN}

La pandemia del SARS-CoV2 COVID-19 que inició en diciembre de 2019 en Wuhan (Hubei, China) (Chen et al., 2020) se extendió rápidamente a otras regiones del mundo, lo que generó tensión, preocupación y miedo entre la población ante la posibilidad de contagiarse y enfermarse gravemente 0 inclusive morir. La mayoría de los países, mediante sus sistemas sanitarios, implementaron medidas para evitar frenar la velocidad de transmisión. Una de ellas fue el confinamiento, el cual tuvo como consecuencia grandes cambios en las sociedades. Las actividades presenciales, laborales, académicas y sociales se suspendieron y se empezaron a desarrollar sistemas de comunicación remota para continuar con los planes y con la productividad; sin embargo, la economía se afectó porque se perdieron miles de empleos (Comisión Económica para América Latina y el Caribe, 2020).

Algunas áreas del conocimiento, como la Psicología y la Salud Mental, han estudiado el impacto de los fenómenos que han perturbado a la humanidad, entre ellos se encuentran los desastres naturales y los generados por el hombre. La pandemia del 2020 también es un fenómeno perturbador para las personas quienes han experimenten miedo, enojo, frustración, tensión, ansiedad y depresión (Pfefferbaum \& North, 2020; Ozamiz-Etxebarria et al., 2020).

Los estudios sobre Salud Mental en diferentes grupos poblacionales señalan la importancia que tiene la vulnerabilidad de quienes padecen enfermedades médicas, como por ejemplo enfermedades metabólicas o cardiovasculares, o psiquiátricas, como en la esquizofrenia, tratornos por dependencia a alcohol y sustancias, y que por distintas razones interrumpen sus tratamientos, carecen de seguimiento y tienen limitada atención especializada. En estas condiciones los cuadros clínicos pueden reactivarse 0 agudizarse (Shigemura et al., 2020).

Los resultados de una encuesta on line en la que participaron mil 210 individuos de población abierta de 194 ciudades en China señalan que en el $53.8 \%$ el impacto psicológico del brote de coronavirus fue de moderado a severo; $16.5 \%$ presentaron síntomas depresivos moderados a severos, $28.8 \%$ tuvieron síntomas de ansiedad moderada a severa y $8.1 \%$ mostró un nivel de estrés moderado a severo (Wang et al., 2020).

Los estudios sobre brotes epidémicos generalmente reportan consecuencias negativas asociadas con el confinamiento y su impacto en la Salud Mental; sin embargo, también es necesario mencionar que hay consecuencias positivas, como se observa en los resultados de una encuesta realizada en familias de Hong Kong al finalizar 


\section{LA PSICOLOGÍA ANTE RETOS DE FUTURO. ABRIENDO CAMINOS}

el confinamiento por el SARS- CoV: 60\% reportaron preocupación por los sentimientos de sus familiares, dos tercios prestaron más atención en su salud mental, y entre 35 y 40\% invirtieron más tiempo en hacer ejercicio y en descansar (Lau et al., 2006).

Otras investigaciones han evaluado las respuestas emocionales, analizando el contenido de los mensajes en las redes sociales 0 a través de encuestas en línea en las que se preguntó, durante el momento del brote, acerca de sus emociones ante la pandemia de COVID-19. Un ejemplo son los usuarios de twitter que recibían información de diferentes fuentes, lo que les generaba una gama de emociones positivas y negativas: miedo, preocupación, malestar, ira, discursos de odio, esperanza, alegría, frustración, falta de algo, aburrimiento y tristeza debido al encierro; así como agradecimiento, reconocimiento, duelo, luto y humor sarcástico (Akif Mustafa et al., 2020).

La Universidad Nacional Autónoma de México realizó una encuesta en línea a los integrantes de su comunidad, en la que participaron estudiantes de iniciación universitaria, bachillerato, licenciatura y de posgrado, además de los docentes y el personal administrativo durante abril y mayo de 2020, periodo en el que la población se encontraba en confinamiento $(n=13073)$. El objetivo de la encuesta fue conocer las opiniones sobre la epidemia de COVID-19 y sus efectos sociales y psicológicos. Las reacciones emocionales que con mayor frecuencia experimentó la comunidad universitaria fueron preocupación, incertidumbre e inquietud por saber más del problema (INFORME COVID-Opiniones-Universitarios, IISUNAM, 5julio.pdf, s. f.).

Para el estudio de las emociones se han propuesto modelos que refieren seis emociones básicas que se asocian con las expresiones faciales universales: felicidad, sorpresa, asco, ira, tristeza y miedo. Otros autores las estudian desde el punto de vista dimensional y las caracterizan por su valencia, refiriendo el componente negativo 0 desagradable y positivo 0 agradable, y al nivel de activación que es el grado de actividad asociado a la respuesta emocional, que va de un estado de tranquilidad a un estado de excitación (Ekman, 1992; Russell, 1980).

Se ha descrito que las personas en las que predomina la expresión de emociones y pensamientos negativos, cuando enfrentan situaciones adversas, manifiestan rigidez en sus respuestas y se incrementa la experiencia negativa que crece en espiral descendente, lo que reduce la posibilidad de generar emociones y pensamientos positivos que pueden ayudar al enfrentamiento y resolución de la adversidad de forma adaptativa o resiliente.

La resiliencia se define como la capacidad de recuperación ante situaciones críticas. Se ha propuesto que se construye de manera individual y relacional a partir de tres dimensiones: cognitiva, actitudinal y emocional. La primera se refiere a la capacidad de generar planes y estrategias viables para la solución de la situación adversa; la segunda se orienta a la búsqueda de elementos o alternativas de solución para sí mismo y los demás, y la tercera que es más experiencial y predispone a la persona a expresar empatía (Notas de coyuntura del CRIM, 2020).

La psicología positiva propone que las emociones de valencia positiva participan en la construcción de la resiliencia. Fredrickson, (2000) formuló la teoría de ampliación y construcción de las emociones positivas a través de cuatro postulados, en el primero mencionó que estas emociones amplían los repertorios de pensamiento-acción de las personas, el segundo refiere la reducción de las emociones de valencia negativa persistentes, el tercero que estimulan la resiliencia psicológica y el cuarto que contribuye a la construcción de mayor bienestar emocional (Kiken \& Fredrickson, 2017).

En el campo de la educación se ha descrito que los docentes que muestran optimismo y emociones positivas mitigan la vulnerabilidad de los estresores laborales favoreciendo el surgimiento de la resiliencia (García \& Gambarte, 2019). La mayoría de los reportes señalan la importancia de la resiliencia para la estabilidad emocional, el éxito académico en la universidad y también que contribuye en la superación de situaciones adversas de tipo emocional, mental o educativo (Sandín-Esteban \& Sánchez-Martí, 2015).

El docente es un profesional que durante la pandemia tuvo que migrar de una modalidad presencial a una modalidad remota para impartir sus clases. A muchos les generó estrés debido a que no contaban con la preparación suficiente para realizar esta tarea; así lo expresaron en las video cápsulas que la Maestría en Docencia para la Educación Media Superior (MADEMS) de la UNAM realizó con los maestrantes que impartían clases en el nivel medio superior. El objetivo de estas video cápsulas de MADEMS (2020 fue apoyar a la comunidad universitaria 


\section{EMOCIONES DE DOCENTES DE LA EDUCACIÓN MEDIA SUPERIOR ANTE LOS CAMBIOS DEL ENTORNO DURANTE EL CONFINAMIENTO POR EL COVID-19}

en la difícil situación educativa que se estaba viviendo, compartiendo estrategias de enseñanza y evaluación del aprendizaje para impartir clases a distancia. Además, se abordaron otras temáticas: "expresando las vivencias docentes durante la pandemia", "motivación ante este evento", "problemas y retos del futuro educativo" y "¿cómo prepararnos ante el futuro educativo?", entre otras (http://madems.posgrado.unam.mx/eventos/capsulas.html).

De acuerdo con Vera García et al., (2019) las emociones de valencia positiva en los docentes les ayudan a enfrentar los estresores en el desempeño laboral, por lo que el objetivo de este trabajo fue identificar las emociones y reflexiones que experimentaron los profesores de bachillerato que se encontraban cursando, durante el confinamiento, la MADEMS con el propósito de reconocer los componentes emocionales que participan en la construcción de la resiliencia frente a la adversidad.

\section{MATERIAL Y MÉTODOS}

Se hizo un estudio exploratorio de corte transversal y analítico.

\section{Participantes}

La muestra fue conformada por los estudiantes de posgrado de la MADEMS. La encuesta se realizó en línea, en abril de 2020 (un mes después del inicio del confinamiento). La participación fue voluntaria, la invitación fue enviada por correo electrónico y a quienes participaron se les otorgó una constancia. Del total de 279 alumnos inscritos, el 20\% respondió la encuesta.

\section{Procedimiento}

Se elaboró un cuestionario, con preguntas cerradas y abiertas, las primeras fueron dirigidas a obtener datos demográficos y las segundas a explorar las emociones y sentimientos; así como, los cambios percibidos en su comunidad durante la pandemia y sus aportaciones profesionales en el futuro.

Se ha propuesto que los videos son herramientas utilizadas que permiten analizar los estados afectivos mediante la extracción de características, como texto, audio e imágenes (Zhang et al., 2020); por ello, en la encuesta se insertaron tres videos obtenidos de la plataforma YouTube. Su contenido se relacionaba con los cambios en el entorno durante el confinamiento (desplazamiento de animales a las ciudades, reducción de la contaminación atmosférica y de los mares, y otro del canto compartido con la comunidad) (https://www.youtube.com/watch?v=5cDAdG6xi2w, https://www.youtube.com/watch?v=1Z_-2dZ-KZM, https://www.youtube.com/watch?v=qqEfr5v65C8).

Se solicitó a cada participante elegir uno de ellos y que expresaran tres emociones que les hubiera provocado el material elegido.

\section{Análisis de datos}

Los datos demográficos de la muestra se analizaron por medio de frecuencias y porcentajes y los textos se estudiaron utilizando el programa worditout (software gratis) que transforma el texto en nubes de palabras; las más frecuentes las agrupa y se representan con una macrografía. Asimismo, se realizó un análisis bivariado utilizando la Xi2, para comparar las emociones de valencia negativa y positiva respecto al sexo, disciplina académica y edad, y la significancia estadística se evaluó con un valor $p$ menor a 0.05. Los datos se procesaron con el paquete estadístico SPSS versión 20.

Las preguntas con respuesta abierta fueron analizadas desde la construcción de la narrativa. Biglia y Bonet (2009) señalan que con la narrativa "se pretende ofrecer un ejemplo de posibilidades de recrear las realidades, de preformarlas colectivamente desde la convergencia entre la inteligencia, las emociones y la experiencia personal".

\section{RESULTADOS}

Contestaron la encuesta 51 estudiantes que cursaban durante el confinamiento la Maestría en Docencia para la Educación Media Superior. El 65\% fue del sexo femenino y 35\% masculino; la edad mínima de los participan- 
tes fue de 25 y la máxima fue de 61 años, y el $70 \%$ se encontraba en un rango de entre 25 a 35 años. Respecto al estado civil el $60 \%$ era soltero y $65 \%$ sin dependientes económicos. Casi el 30\% de ellos impartían la asignatura de Biología, seguidos de un 16\% de Español; la menor participación se obtuvo de maestrantes que impartían la asignatura de Física en el nivel medio superior (tabla 1).

Tabla 1. Características sociodemográficas

\begin{tabular}{lll}
\hline $\mathbf{n = 5 1}$ & \multicolumn{1}{c}{$\mathbf{n}$} & $\mathbf{\%}$ \\
\hline Sexo & 33 & 64.7 \\
Mujer & 18 & 35.3 \\
Hombre & & \\
\hline Disciplina & & \\
\hline Biología & 15 & 29.4 \\
Ciencias de la Salud & 3 & 5.9 \\
Ciencias Sociales & 3 & 5.9 \\
Español & 8 & 15.7 \\
Filosofía & 3 & 5.9 \\
Física & 1 & 2 \\
Geografía & 3 & 5.9 \\
Ingles & 2 & 3.9 \\
Matemáticas & 5 & 9.8 \\
Psicología & 5 & 9.8 \\
Química & 3 & 5.9 \\
\hline
\end{tabular}

En la figura 1, se observan las palabras más representativas expresadas por los participantes. Las más frecuentes fueron las de valencia positiva contrario de las negativas. La emoción con valencia positiva que se expresó más veces fue "esperanza" y la de negativa fue "tristeza". 


\section{EMOCIONES DE DOCENTES DE LA EDUCACIÓN MEDIA SUPERIOR ANTE LOS CAMBIOS DEL ENTORNO DURANTE EL CONFINAMIENTO POR EL COVID-19}

\section{Figura 1. Nube de palabras "emociones" expresadas}

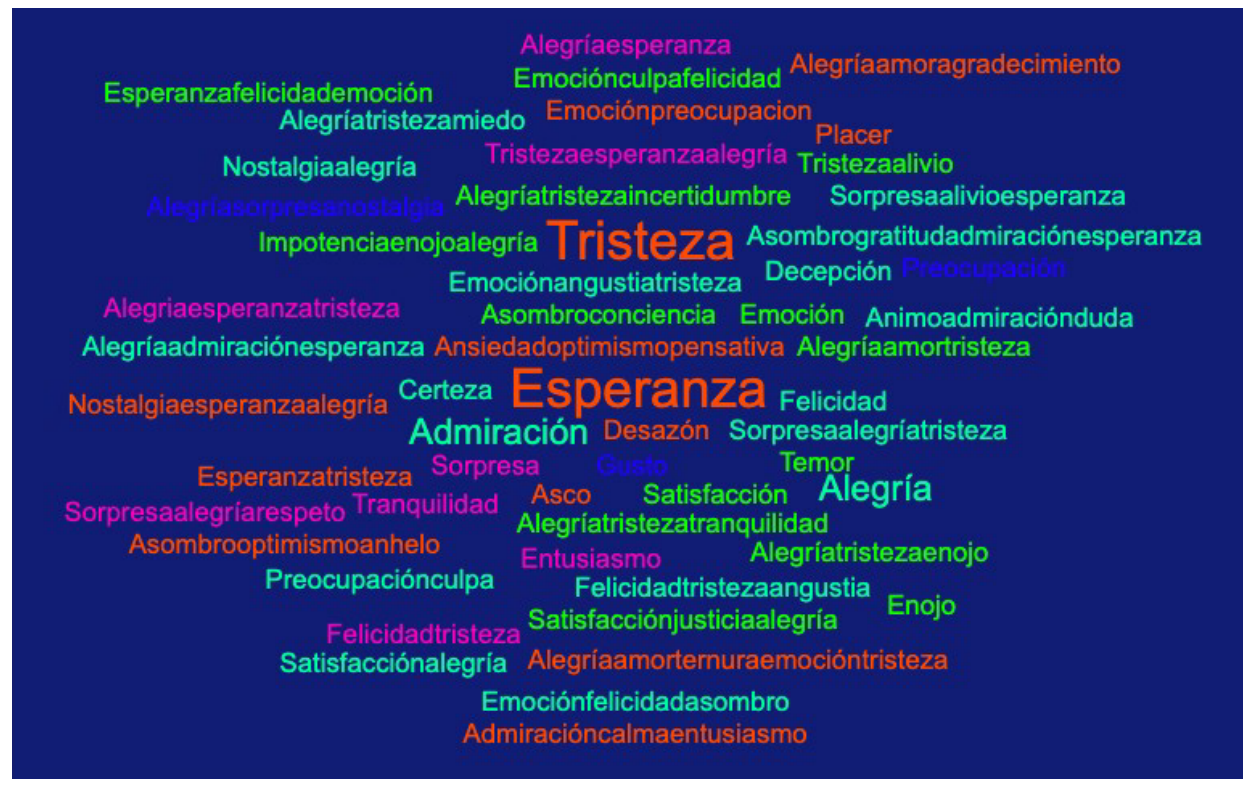

Después de ver el video se analizaron las tres emociones expresadas por los participantes. En un contexto general el $71 \%$ fueron emociones positivas y $29 \%$ negativas. El análisis según la valencia positiva y negativa de la primera y segunda emoción expresadas, arrojaron diferencias significativas de $p \quad$ y $\quad .002$, predominandolas positivas; la tercera no arrojó diferencias entre positivas y negativas (tabla 2).

Tabla 2. Comparación entre las tres respuestas de emociones positivas y negativas

\begin{tabular}{llclll}
\hline & $\boldsymbol{n}$ & $\boldsymbol{x}^{2}$ & $\boldsymbol{g l}$ & $\boldsymbol{p}$ \\
\hline $\begin{array}{l}\text { Positiva } \\
\text { Negativa }\end{array}$ & $\mathbf{1}^{\text {a }}$. Emoción & 38 & 12.25 & 1 & 0.000 \\
Positiva & $\mathbf{2}^{\text {a. }}$ Emoción & 13 & & & \\
Negativa & & 30 & 10 & 1 & 0.002 \\
Positiva & $\mathbf{3}^{\text {a }}$. Emoción & 10 & & & \\
Negativa & & 14 & 0.36 & 1 & 0.549 \\
\hline $\mathbf{p} \geq=\mathbf{0 . 0 5}$ & & 11 & & &
\end{tabular}

Asimismo, se determinó que no existieron diferencias significativas entre el tipo de emoción expresada según el sexo, edad y disciplina académica. 


\section{ANÁLISIS DE LAS PREGUNTAS ABIERTAS}

Las respuestas a la pregunta abierta ¿cuáles han sido los cambios más importantes que visualizas en tu comunidad durante la pandemia?, fueron clasificadas en narraciones con aspectos de impacto positivo con un $55 \%$ y negativo $45 \%$. Al categorizar las respuestas positivas, en su mayoría involucraban aspectos de "cuidado de medio ambiente y contaminación".

"Pudimos parar y relajarnos un poco, ante la vida tan rápida que vivimos; sobre todo, darnos cuenta de los beneficios ambientales".

Las otras categorías con significado fueron: la conciencia social, el fortalecimiento de las relaciones familiares; así como, la adquisición de nuevos conocimientos, el uso de las Tecnologías de la Información y la Comunicación (TIC) en la enseñanza y los cuidados de salud e higiene.

Las narrativas con aspectos negativos versaron sobre las categorías: aspectos económicos, de educación entre las que resaltaron la ignorancia, por ejemplo, no creer que existe el virus, los ataques al personal de salud y no utilizar cubre bocas; así como, narraciones de indiferencia, poca solidaridad, aislamiento social, problemas de salud mental y con vecinos y familiares.

"En primer lugar el aislamiento voluntario que es funcional para los estratos sociales más favorecidos, debido a que las personas más pobres o de trabajos de servicios alimenticios o de limpieza tienen que seguir exponiéndose al contacto con personas. También se tiene una batalla con la ignorancia debido que se agrede al personal de salud en algunas localidades, pues la gente cree que este personal los puede contagiar".

Otra de las preguntas de respuesta abierta fue: Después de que pase la pandemia, ¿desde tu campo de conocimiento qué cambios contundentes realizarías en la forma de vida de tu comunidad, país o en el mundo? Esta pregunta fue contestada por los maestrantes sin visualización del futuro, consideramos incluirlas ya que sólo la percibieron como una continuidad de mejora de lo difundido por las noticias o bien del contexto actual, las categorías más frecuentemente expresadas tuvieron una connotación positiva y fueron alrededor de cambios sociales y en el sector salud.

"Cuando termine la pandemia habrá cambios en el sistema de salud de todos los países del mundo, porque como es bien sabido, en muchos lugares han colapsado y han debido habilitar hoteles como hospitales y auditorios o pistas de hielo como morgues. Aunque respetados y bien remunerados, los médicos exigirán mejores condiciones de trabajo y en la búsqueda de sus demandas, se generarán muchos cambios sociales en diferentes ámbitos".

Seguidas por las respuestas en las temáticas, del cuidado del medio ambiente y la promoción de la educación en línea y a distancia.

"Creo que el trabajo en línea se convertirá en algo que muchas comunidades aplicarán en su forma de trabajo".

\section{DISCUSIÓN}

En este estudio se observó que ante los cambios en el entorno durante el confinamiento por la epidemia del COVID-19, los estudiantes de posgrado que cursaban la Maestría en Docencia para el Nivel Medio Superior expresaron, en mayor proporción, las emociones positivas. Las representaciones de las emociones positivas experimentadas por los docentes durante el confinamiento fueron amor, empatía, optimismo y compasión, las emociones negativas estuvieron representadas por enojo, miedo y tristeza.

Las emociones positivas son elementos fundamentales para la construcción de la resiliencia y el bienestar de las personas, lo que favorece la capacidad de afrontar situaciones de crisis, que generan estrés y problemas de Salud Mental. (Fredrickson., et al.2000; de Vera et al.,2019)

Las respuestas emocionales y reflexiones positivas de los docentes que participaron en el estudio pueden explicarse por el ambiente que se generó en este posgrado, en el cual se mantuvo la comunicación y se sintieron acompañados, tanto por los profesores y tutores, como por sus compañeros, ya que los propios alumnos generaron materiales de apoyo en línea mostrando su aplicación para el desarrollo de los contenidos temáticos y para 


\section{EMOCIONES DE DOCENTES DE LA EDUCACIÓN MEDIA SUPERIOR ANTE LOS CAMBIOS DEL ENTORNO DURANTE EL CONFINAMIENTO POR EL COVID-19}

cumplir con su trabajo docente. Algunos de ellos, expresaron que la pandemia tuvo consecuencias en su práctica docente. En pocas semanas la mayoría de las actividades, tanto en escuelas como en universidades, pasaron de la modalidad presencial a on line. Algunos docentes lo percibieron como una oportunidad de adquirir nuevas habilidades y conocimientos, otros como una adversidad que les generó estrés por el desconocimiento del uso de las TIC en la enseñanza.

La mayoría de los docentes expresaron que implementaron cambios en su estilo de vida durante el distanciamiento social. Paradójicamente, la mayoría lo hizo fortaleciendo sus relaciones interpersonales más significativas con hijos, pareja, amigos y padres, no sólo disminuyendo la distancia social, sino estrechando vínculos frente a la adversidad.

Finalmente, los resultados demuestran que ante el confinamiento e incertidumbre, los estudiantes que se están formando en el programa MADEMS para obtener una formación docente más sólida, han desarrollado "la resiliencia del docente", la cual genera una acción pedagógica en los estudiantes que, a pesar de la adversidad, se presenta como una herramienta clave para generar climas emocionalmente positivos y más seguros para el aprendizaje (Vicente de Vera, García \& Gabari, Gambarte, 2019), lo que también tiene como consecuencia lo expresado por Serrano y Sanz (2019) quienes señalan que cuando el docente se percibe a sí mismo como poseedor de cierto grado de habilidades para regular sus emociones, utiliza más estrategias activas para enfrentar situaciones estresantes en el entorno académico.

\section{ALCANCES/ LIMITACIONES}

Como parte de la formación académica de los docentes, es necesario desarrollar programas que promuevan el reconocimiento de emociones positivas que integren la construcción de la resiliencia, ya que esta puede verse reflejada en las conductas y desempeño con los jóvenes estudiantes de enseñanza media superior que se encuentran en una etapa del desarrollo crucial como la adolescencia, en la que se consolidan procesos psicológicos y sociales que contribuyen en la transición a la vida adulta. Una de las limitaciones de este estudio exploratorio fue que no se utilizaron escalas para evaluar la valencia y el nivel de activación de las emociones, así como de la medición de la resiliencia.

\section{CONCLUSIONES}

Los profesores inscritos en esta Maestría mostraron emociones positivas ante la crisis generada por el COVID-19, que pudieron estar asociadas a la construcción de la resiliencia que se ve reflejada en su labor y que les ayuda a tener una perspectiva constructiva a pesar de las contingencias o adversidades, así como en la interacción con los alumnos de enseñanza media superior y su capacidad para adaptarse ante los cambios inesperados, implementando estrategias y materiales en línea para continuar su trabajo. Esto nos indica la gran necesidad de formar a los profesores en el uso de las TIC en la educación, ya que de lo contrario se corre el riesgo de que los profesores enfrenten una condición de estrés agudo que pueda desencadenar trastornos de ansiedad y depresión que cuando son severos se experimentan como una condición caótica que los desorganiza en su funcionamiento cognitivo y afectivo dando como resultado una desadaptación laboral y académica que les impide cumplir con sus labores.

Otros aspectos que se observaron en los docentes fueron el fortalecimiento de las relaciones interpersonales y familiares, el interés por los cambios medioambientales, así como ver a la

crisis como una oportunidad de crecimiento personal y profesional. Asimismo, ante los resultados obtenidos en este trabajo, concluimos que es necesario implementar en la formación docente asignaturas derivadas de los nuevos hallazgos de las neurociencias de la educación que fortalezcan el manejo de las emociones ante acontecimientos adversos como la pandemia o bien ante desastres naturales, lo que sin duda redundará en el desarrollo de la resiliencia. 


\section{REFERENCIAS BIBLIOGRÁFICAS}

Akif Mustafa, Imaduddin Ansari, Subham Kumar Mohanta, y Shalem Balla. «Public reaction to COVID-19 on Twitter: a thematic analysis». EPRA International Journal of Multidisciplinary Research (IJMR), 25 de mayo de 2020, 349-58. https://doi.org/10.36713/epra4518.

Biglia, Barbara, y Jordi Bonet i Martí. «La construcción de narrativas como método de investigación psico-social. Prácticas de escritura compartida». Forum: Qualitative Social Research 10, n. ${ }^{0}$ (2009). https://ddd.uab.cat/record/146075.

Caribe, Comisión Económica para América Latina y el. América Latina y el Caribe ante la pandemia del COVID19: efectos económicos y sociales. CEPAL, 2020. https://www.cepal.org/es/publicaciones/45337-americalatina-caribe-la-pandemia- covid-19-efectos-economicos-sociales.

—_. «América Latina y el Caribe ante la pandemia del COVID-19: efectos económicos y sociales». CEPAL, 3 de abril de 2020. https://www.cepal.org/es/publicaciones/45337-america-latina-caribe-la-pandemiacovid-19-efectos-economicos-sociales.

Chen, Qiongni, Mining Liang, Yamin Li, Jincai Guo, Dongxue Fei, Ling Wang, Li He, et al. «Mental Health Care for Medical Staff in China during the COVID-19 Outbreak». The Lancet. Psychiatry 7, n. ${ }^{0}$ (2020): e15-16. https://doi.org/10.1016/S2215-0366(20)30078-X.

Claudia Infante, Peláez Ballestas, y Murillo López. «Universitarios opinan sobre la COVID-19 y sus efectos sociales. Comisión UNAM COVID-19», 4 de julio de 2020. https://covid19comisionunam.unamglobal.com/?p=87268.

Ekman, Paul. «An argument for basic emotions». Cognition and Emotion 6, n. 0 3-4 (1 de mayo de 1992): 169200. https://doi.org/10.1080/02699939208411068.

Kiken, Laura G., y Barbara L. Fredrickson. «Cognitive aspects of positive emotions: A broader view for wellbeing». En The happy mind: Cognitive contributions to well- being, 157-75. Cham, Switzerland: Springer International Publishing, 2017. https://doi.org/10.1007/978-3-319-58763-9_9.

Lau, Joseph T. F., Xilin Yang, H. Y. Tsui, Ellie Pang, y Yun Kwok Wing. «Positive mental health-related impacts of the SARS epidemic on the general public in Hong Kong and their associations with other negative impacts». Journal of Infection 53, n. ${ }^{0} 2$ (1 de agosto de 2006): 114-24. https://doi.org/10.1016/j.jinf.2005.10.019.

Mehrad, Aida. «Post-traumatic stress disorder (PTSD) effect of coronavirus (COVID-19) pandemic and role of emotional intelligence». Journal Of Social Science Research 15 (22 de mayo de 2020): 185-90. https://doi.org/10.24297/jssr.v15i.8750.

Ozamiz-Etxebarria, Naiara, Maria Dosil-Santamaria, Maitane Picaza-Gorrochategui, y Nahia Idoiaga-Mondragon. «Stress, anxiety, and depression levels in the initial stage of the COVID-19 outbreak in a population sample in the northern spain». Cadernos De Saude Publica 36, n. ${ }^{0} 4$ (2020): e00054020. https://doi.org/10.1590/0102- 311X00054020.

Pfefferbaum, Betty, y Carol S. North. «Mental health and the COVID-19 pandemic». New England Journal of Medicine, 13 de abril de 2020, NEJMp2008017. https://doi.org/10.1056/NEJMp2008017.

_ _. «ental health and the COVID-19 pandemic». New England Journal of Medicine, 13 de abril de 2020, NEJMp2008017. https://doi.org/10.1056/NEJMp2008017.

_ . «Mental health and the COVID-19 pandemic». The New England Journal of Medicine 383, n. ${ }^{0} 6$ (6 de agosto de 2020): 510-12. https://doi.org/10.1056/NEJMp2008017.

Román, Fabián, Anna Forés, Ismael Calandri, Rosalba Gautreaux, Alejandro Antúnez, Dalul Ordehi, Lenin Calle, et al. «Resiliencia de docentes en distanciamiento social preventivo obligatorio durante la pandemia de COVID-19». Journal of Neuroeducation 1, n. ${ }^{0} 1$ (15 de julio de 2020): 76-87. https://doi.org/10.1344/joned.v1i1.31727.

Russell, James A. «A circumplex model of affect.» Journal of Personality and Social Psychology 39, n. ${ }^{0} 6$ (1980): 1161-78. https://doi.org/10.1037/h0077714. 


\section{EMOCIONES DE DOCENTES DE LA EDUCACIÓN MEDIA SUPERIOR ANTE LOS CAMBIOS DEL ENTORNO DURANTE EL CONFINAMIENTO POR EL COVID-19}

- «A circumplex model of affect». Journal of Personality and Social Psychology 39, n. ${ }^{0} 6$ (1980): 116178. https://doi.org/10.1037/h0077714.

. «A circumplex model of affect.» Journal of Personality and Social Psychology 39, n. 0 (1980): 116178. https://doi.org/10.1037/h0077714.

Salazar, Karla. «Notas de coyuntura del CRIM». Universidad Nacional Autónoma de México, Centro Regional de Investigaciones Multidisciplinarias, 2020. https://doi.org/10.22201/crim.001r.2020.

Sánchez-Villena, Andy Rick, y Valeria de La Fuente-Figuerola. «COVID-19: cuarentena, aislamiento, distanciamiento social y confinamiento, ¿son lo mismo?» Anales De Pediatria (Barcelona, Spain: 2003) 93, n. ${ }^{0} 1$ (julio de 2020): 73-74. https://doi.org/10.1016/j.anpedi.2020.05.001.

Sandín-Esteban, María-Paz, y Angelina Sánchez-Martí. «Resilience and school success of young immigrants / Resiliencia y éxito escolar en jóvenes inmigrantes». Journal for the Study of Education and Development 38, n. ${ }^{1}$ (2 de enero de 2015): 175-211. https://doi.org/10.1080/02103702.2015.1009232.

Sarmiento, Angela Serrano, y Roberto Sanz Ponce. «Reflexiones y propuestas prácticas para desarrollar la capacidad de resiliencia frente a los conflictos en la escuela». Publicaciones: Facultad de Educación y Humanidades del Campus de Melilla 49, n. ${ }^{0}$ Extra 1 (Número especial) (2019): 177-90.

—. «Reflexiones y propuestas prácticas para desarrollar la capacidad de resiliencia frente a los conflictos en la escuela». Publicaciones: Facultad de Educación y Humanidades del Campus de Melilla 49, n. ${ }^{0}$ Extra 1 (Número especial) (2019): 177-90.

Shigemura, Jun, Robert J. Ursano, Joshua C. Morganstein, Mie Kurosawa, y David M. Benedek. «Public responses to the novel 2019 Coronavirus (2019-NCoV) in Japan: Mental health consequences and target populations». Psychiatry and Clinical Neurosciences 74, n. 04 (2020): 281-82. https://doi.org/10.1111/pcn.12988.

Vicente de Vera,García, M. Inmaculada Vicente de Vera, y M. Ines Gabari Gabari, Gambarte. «Emociones positivas: una herramienta psicológica que contribuye al proceso de resiliencia en los profesionales de la educación». Revista INFAD de Psicología. International Journal of Developmental and Educational Psychology. 4, n. ${ }^{0} 1$ (31 de agosto de 2019): 159-72. https://doi.org/10.17060/ijodaep.2019.n1.v4.1539.

Wang, Cuiyan, Riyu Pan, Xiaoyang Wan, Yilin Tan, Linkang Xu, Cyrus S. Ho, y Roger C. Ho. «Immediate psychological responses and associated factors during the initial stage of the 2019 coronavirus disease (COVID-19) epidemic among the general

population in China». International Journal of Environmental Research and Public Health 17, n. 05 (enero de 2020): 1729. https://doi.org/10.3390/ijerph17051729.

Zhang, Jing, Xingyu Wen, y Mincheol Whang. «Recognition of emotion according to the physical elements of the video». Sensors 20, n. ${ }^{0} 3$ (enero de 2020): 649. https://doi.org/10.3390/s20030649. 